\title{
A sífilis congênita no olhar da enfermagem
}

\author{
Congenital syphilis as seen by nursing \\ La sífilis congénita en la visión de la enfermería
}

\author{
Valéria Silva de Mello; Rosângela da Silva Santos ${ }^{I I}$
}

\begin{abstract}
RESUMO: Revisão integrativa que objetivou discutir publicações científicas relacionadas à sífilis congênita e à enfermagem. A questão norteadora foi: Que aspectos norteiam a assistência da enfermeira à gestante, em relação à prevenção e controle da Sífilis Congênita? Dados coletados de abril a maio de 2015, nas bases de dados Literatura Latino-Americana e do Caribe em Ciências da Saúde, Medical Literature Analysis and Retrieval Sistem On-Linee Scientific Eletronic Library Online, com os descritores sífilis congênita e enfermagem, nos idiomas português e inglês. Selecionaram-se oito publicações na íntegra, do período de 2005 a 2014. Emergiram três categorias: fatores de risco da sífilis congênita; inadequação da assistência prestada e dificuldades dos profissionais de saúde na prevenção e controle da sífilis. A prevenção e o controle da sífilis na gestação e sífilis congênita continuam um desafio para o Ministério da Saúde. Os resultados poderão ajudar na melhoria da qualidade da atuação do profissional. Palavras-Chave: Sífilis congênita; enfermagem de atenção primária; gestantes; cuidado pré-natal.
\end{abstract}

ABSTRACT: This integrative review discussed scientific publications relating to congenital syphilis and to nursing. The guiding question was: What factors guide nursing care for pregnant women as regards preventing and controlling congenital syphilis? Data were collected from April to May 2015 from the following databases: Latin American and Caribbean Health Sciences Literature, Medical Literature Analysis and Retrieval System Online and Scientific Electronic Library Online, with the descriptors 'congenital syphilis' and 'nursing', in Portuguese and English. Eight full publications were selected, from the period 2005 to 2014. Three categories emerged: risk factors for congenital syphilis; inappropriateness of the care provided; and health staffs' difficulties in syphilis prevention and control. Prevention and control of syphilis in pregnancy and congenital syphilis remain a challenge for the Ministry of Health. The study results may help improve the quality of health personnel's work.

Keywords: Congenital syphilis; primary care nursing; pregnant women; antenatal care.

RESUMEN: Revisión integradora cuyo objetivo fue discutir publicaciones científicas relacionadas con la sífilis congénita y la enfermería. La pregunta orientadora fue: ¿Qué aspectos guían la atención de la enfermería a las mujeres embarazadas, en cuanto a la prevención y el control de la sífilis congénita? Fueron obtenidos datos de abril a mayo de 2015, de las bases de datos Literatura Latinoamericana y del Caribe en Ciencias de la Salud, Medical Literature Analysis and Retrieval Sistem Online y Scientific Electronic Library Online; las palabras clave fueron sífilis congénita y enfermería, en portugués e inglés. Se han seleccionado ocho publicaciones en su totalidad, del período de 2005 a 2014. Destacaron tres categorías: factores de riesgo de la sífilis congénita; inadecuación de la atención prestada y dificultades de los profesionales de salud en la prevención y control de la sífilis congénita. La prevención y el control de la sífilis durante el embarazo y la sífilis congénita siguen siendo un desafío para el Ministerio de Salud. Los resultados podrán ayudar a mejorar la calidad del desempeño profesional. Palabras Clave: Sífilis congénita; enfermería de atención primaria; embarazadas; atención prenatal.

\section{INTRODUÇÃO}

A sífilis é uma doença infecciosa, que desafia a humanidade há séculos. Tornou-se conhecida na Europa no século XV, quando irrompeu uma epidemia muito grave de sífilis. A rápida disseminação por todo o continente transformou-a numa das principais pragas mundiais ${ }^{1}$.

Apesar de não ocupar espaço importante na mortalidade materna, apresenta alta prevalência e facilidade de transmissão vertical. No ciclo gravídico puerperal, a infecção é preocupante, devido ao agravo na forma congênita, incrementando os números de mortalidade perinatal, abortos e sequelas neurológicas em recém-nascidos ${ }^{2}$.

A sífilis na gestação e a sífilis congênita atingiram proporções não justificadas diante da disponibilidade de diagnóstico e tratamento, assim como seus números não traduzem o resultado esperado das constantes campanhas do Ministério da Saúde para o combate à doença. É ainda um indicador de qualidade de atenção pré-natal, pois quando este é realizado corretamente, não há motivos que justifiquem o nascimento de crianças com sífilis ${ }^{3}$.

Neste contexto, traçou-se a seguinte questão norteadora: Que aspectos norteiam a assistência da enfermeira à gestante, em relação à prevenção e controle da sífilis congênita? Para responder a esta questão, realizou-se uma revisão integrativa com o seguinte objetivo: discutir publicações científicas relacionadas à sífilis congênita e a enfermagem.

IEnfermeira. Servidora da Prefeitura Municipal do Rio de Janeiro, lotada no Hospital Maternidade Fernando Magalhães. Mestranda da Faculdade de Enfermagem da Universidade do Estado do Rio de Janeiro. Brasil. E-mail: valsilmel@gmail.com.

IIDoutora em Enfermagem. Professora Adjunta da Faculdade de Enfermagem da Universidade do Estado do Rio de Janeiro. Pesquisadora 1C Conselho Nacional de Pesquisa. Fundação de Amparo à Pesquisa do Rio de Janeiro. Brasil. E-mail: rosangelaufrj@gmail.com. 


\section{REVISÃO DE LITERATURA}

A origem da sífilis é controversa. A primeira teoria sustenta que a doença era endêmica na América e que teria sido levada para a Europa pelos marinheiros de Cristóvão Colombo. A segunda teoria, a do Velho Mundo, apoia que as treponematoses já existiam em território europeu e adquiriram um aumento e diferenciação permitindo a transmissão sexual e desencadeando epidemias ${ }^{4}$.

A sífilis é causada pela bactéria Treponema pallidum e acomete, praticamente, todos os órgãos e sistemas. As principais formas de transmissão são a via sexual (adquirida) e a via vertical (congênita), da mãe para o feto ${ }^{5}$.

A proposta do Ministério da Saúde era de eliminar a sífilis congênita até o ano 2000. A meta não foi alcançada e continua-se a enfrentar a crescente incidência da sífilis congênita na população. A notificação de casos de sífilis congênita no Brasil aumentou em 34\% entre os anos de 2010 e 2011. A região que apresenta a maior taxa de incidência de sífilis congênita é a Nordeste. Entre os estados, a maior incidência é no Rio de Janeiro ${ }^{6}$.

Essa problemática tem mobilizado não só o Brasil, mas também a comunidade internacional. A Organização Pan-Americana da Saúde (OPAS) e o Fundo das Nações Unidas para a Infância (UNICEF), em 2009, lançaram a iniciativa regional para a eliminação da transmissão vertical do HIV e da sífilis na América Latina e Caribe. Em 2010, essa iniciativa regional articulou-se ao Plano Global para eliminação de novas infecções pelo HIV entre crianças até 2015 e manter suas mães vivas. Nesse mesmo ano, os estados-membros da OPAS aprovaram o Plano de ação para a eliminação da transmissão vertical do HIV e da sífilis congênita, estabelecendo metas para o ano de 2015 de redução da taxa de transmissão do HIV e da taxa de incidência de sífilis congênita.

No Brasil, o Sistema de Vigilância Epidemiológica e de Notificação Compulsória de Doenças foi criado em 1976, pelo Decreto Presidencial no 78.231. Em 1986, a sífilis congênita tornou-se doença de notificação compulsória nacional por meio da Portaria n ${ }^{\circ} 542$ de 22 de dezembro; a sífilis em gestante passou a integrar o Sistema Nacional de Vigilância em 2005. Em 2014, foi publicada a Portaria $\mathrm{n}^{\mathrm{o}} 1.271$, que incluiu também a notificação da sífilis adquirida?

Em 2011, foi instituída a Rede Cegonha, visando assegurar à mulher e à criança uma rede de cuidados. Entre suas ações estão a prevenção e tratamento das infecções sexualmente transmissíveis (ISTs), com disponibilização de testes rápidos de sífilis e HIV?

\section{Metodologia}

A revisão integrativa da literatura consiste num levantamento sistemático de publicações sobre determinado assunto, proporcionando sintetizar o conhe- cimento e incorporar a aplicação dos resultados dos estudos realizados ${ }^{8}$. O propósito é obter entendimento de um determinado fenômeno baseando-se em estudos anteriores. Permite a avaliação crítica e a síntese das evidências disponíveis do tema investigado. Proporciona a atualização sobre $\mathrm{o}$ assunto utilizando dados relevantes em diferentes momentos e lugares. $O$ produto final é o conhecimento atual do tema investigado, identificando lacunas que direcionarão o desenvolvimento de pesquisas futuras ${ }^{9}$. É composta de seis fases: identificação do tema; busca na literatura; definições das informações; categorização dos estudos; análise dos resultados e síntese do conhecimento ${ }^{10}$.

Realizou-se uma busca nos meses de abril e maio de 2015 nas seguintes bases de dados: Scientific Eletronic Library Online (SCIELO), Literatura Latino-Americana e do Caribe em Ciências da Saúde (LILACS) e Medical Literature Analysis and Retrieval Sistem on-line (MEDLINE). Foram utilizados os seguintes descritores e suas combinações nas línguas portuguesa e inglesa: sífilis congênita and enfermagem. Os critérios de inclusão definidos para a seleção dos artigos foram: publicações em português e inglês; artigos na íntegra que retratassem a temática da sífilis congênita e sua relação com a enfermagem e publicações indexadas no período de 2005 a 2014.

Ao iniciar a busca, foram encontrados 32 estudos: oito artigos na LILACS, 20 na MEDLINE e quatro artigos na SCIELO. Aplicando-se o recorte temporal de 2005 a 2014, reduziram-se para: seis artigos na LILACS, quatro na MEDLINE e três na SCIELO. Verificou-se que cinco artigos não tinham relação com os objetivos do estudo e foram excluídos. A seleção final da amostra totalizou oito artigos: quatro na LILACS, um na MEDLINE e três na SCIELO. Ver Figura 1.

Construiu-se um instrumento para coleta de dados com as seguintes informações: título, autores, ano, publicação, país, idioma, participantes e tipo de estudo. Aos oito artigos ${ }^{11-18}$, foram atribuídos a identificação de A1 a A8 para facilitação e organização dos dados encontrados.

$N^{\circ}$ de artigos obtidos: 32

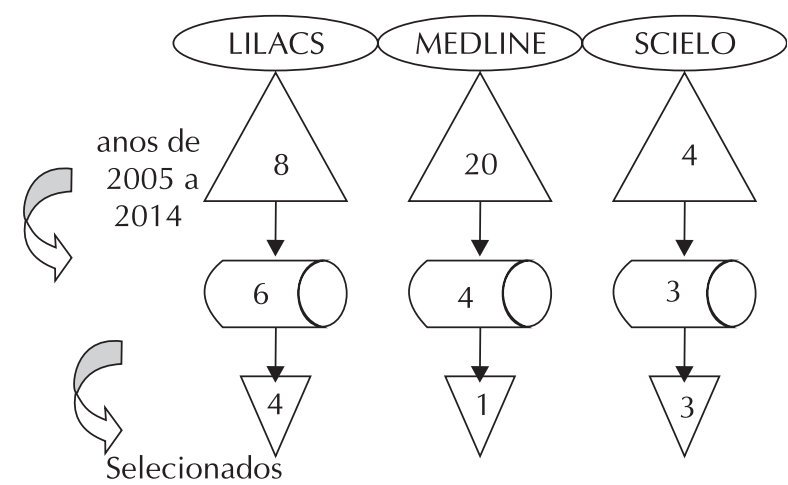

Total $=\mathbf{8}$

FIGURA 1: Fluxograma da busca dos artigos para revisão. 


\section{Resultados e Discussão}

Iniciou-se a fase de análise temática mediante leitura na íntegra de todos os artigos ${ }^{11-18}$, seguida da identificação dos aspectos necessários para a catego- rização. Ver Figura 2. Os resultados encontrados nas publicações foram delimitados em três categorias: Fatores de risco da sífilis congênita; Assistência de enfermagem prestada à gestante; e Dificuldades profissionais na prevenção e controle da sífilis.

\begin{tabular}{|c|c|c|c|c|c|}
\hline Artigo & Título & Ano & Idioma & Participantes & Tipo de estudo \\
\hline A1 & $\begin{array}{l}\text { Sífilis congênita no Rio Grande do } \\
\text { Norte: estudo descritivo do perío- } \\
\text { do } 2007-2010^{11}\end{array}$ & 2014 & Português & $\begin{array}{l}\text { Sistema de Informações sobre } \\
\text { Nascidos Vivos (SINASC) e Sis- } \\
\text { tema de Informações de Agravo } \\
\text { e de Notificação (SINAN) }\end{array}$ & Estudo descritivo \\
\hline A2 & $\begin{array}{l}\text { Complicações da sífilis congênita: } \\
\text { uma revisão de literatura }{ }^{12}\end{array}$ & 2014 & Português & $\begin{array}{l}\text { Bases de dados LILACS, MED- } \\
\text { LINE e SCIELO, de março a } \\
\text { junho de } 2012 \text {. }\end{array}$ & $\begin{array}{l}\text { Estudo descritivo } \\
\text { e bibliográfico }\end{array}$ \\
\hline A3 & $\begin{array}{l}\text { Análise dos Casos de Sífilis Congê- } \\
\text { nita em Sobral, Ceará: Contribui- } \\
\text { ções para Assistência Pré-Natal }{ }^{13}\end{array}$ & 2012 & Português & $\begin{array}{l}\text { Nove casos de sífilis congênita } \\
\text { no Município de Sobral em } \\
2010 .\end{array}$ & $\begin{array}{l}\text { Estudo descritivo, } \\
\text { documental e } \\
\text { exploratório }\end{array}$ \\
\hline A4 & $\begin{array}{l}\text { Sífilis congênita no Ceará: análise } \\
\text { epidemiológica de uma década }{ }^{14}\end{array}$ & 2013 & Português & $\begin{array}{l}\text { Casos de sífilis congênita no- } \\
\text { tificados no SINAN, no perío- } \\
\text { do de } 2000 \text { a } 2009 . \mathrm{N}=2.930\end{array}$ & $\begin{array}{l}\text { Estudo transversal e } \\
\text { documental. }\end{array}$ \\
\hline A5 & $\begin{array}{l}\text { Reação de Jarisch-Herxheimer em } \\
\text { Gestante com sífilis: relato de } \\
\text { caso }^{15} \text {. }\end{array}$ & 2011 & Português & $\begin{array}{l}\text { Gestante que apresentou Reação } \\
\text { de Jarisch-Herxheimer. }\end{array}$ & Estudo descritivo \\
\hline A6 & $\begin{array}{l}\text { Resurgence of congenital syphilis: } \\
\text { diagnosis and treatment }{ }^{16}\end{array}$ & 2011 & Inglês & Criança com sífilis congênita & Estudo descritivo \\
\hline A7 & $\begin{array}{l}\text { Manejo da sífilis na gestação: co- } \\
\text { nhecimentos, práticas e atitudes dos } \\
\text { profissionais pré-natalistas da rede } \\
\text { SUS do Município do RJ }{ }^{17}\end{array}$ & 2013 & Português & $\begin{array}{l}102 \text { Profissionais pré-natalistas } \\
\text { da rede SUS do Município do RJ }\end{array}$ & Estudo transversal \\
\hline A8 & $\begin{array}{l}\text { Knowledge of health care profes- } \\
\text { sionals regarding the vertical } \\
\text { transmission of syphilis in Fortaleza } \\
-\mathrm{CE}, \text { Brazil }^{18}\end{array}$ & 2014 & Inglês & $\begin{array}{l}269 \text { profissionais da estratégia } \\
\text { saúde da família - Fortaleza }\end{array}$ & Estudo transversal \\
\hline
\end{tabular}

FIGURA 2: Identificação dos artigos selecionados da Literatura sobre sífilis congênita. Período 2011-2014.

\section{Fatores de risco da sífilis congênita}

Dos oito artigos analisados, cinco evidenciam que as gestantes com sífilis possuem baixo nível social, pouca ou nenhuma escolaridade ${ }^{11-14,17}$. Um dos estudos ${ }^{14}$ constata, em sua amostragem, que mais da metade é analfabeta.

Outro estudo sobre o perfil epidemiológico da sífilis congênita, no Município de Natal, evidenciou que mães pertencentes a estratos sociais menos favorecidos contam com pouco acesso às informações necessárias para evitar a infecção e consequente transmissão vertical da sífilis; ao diagnóstico da doença, desconhecem a importância de um tratamento completo e eficaz. Fatores socioeconômicos, como baixa escolaridade e baixa renda familiar são determinantes de inúmeros agravos em saúde pública. Mães sem instrução e mães cujas famílias são de baixa renda têm maior risco de terminarem uma gestação com o óbito fetal do que mães que estão acima desses patamares ${ }^{19}$.
A precocidade sexual, sexo desprotegido, o aumento de mães adolescentes, uso de drogas ilícitas, tabagismo e etilismo são discutidos em duas pesquisas $^{13,14}$ realizadas no Ceará sobre o perfil epidemiológico da sífilis congênita, naquele estado. Tais estudos constatam que tais fatores estão presentes na maioria dos casos ${ }^{13,14}$. Estes fatores são também enfatizados em outro estudo de revisão bibliográfica ${ }^{12}$.

Um trabalho de conclusão de curso, realizado em Minas Gerais, em estratégia saúde da família ${ }^{20}$, corrobora esses resultados e ratifica que o início da vida sexual dos jovens ocorre cada vez mais precocemente de forma não adequada, não programada, não planejada e quase sempre de maneira insegura. Muitas adolescentes não têm consciência da gravidade das ISTs e de suas consequências na concepção. $O$ não uso do preservativo ou seu uso inadequado podem acarretar não só infecção por doenças sexualmente transmissíveis como provocar gravidez indesejada ${ }^{20}$. 
Evidenciou-se, nesta revisão integrativa, como fator de risco para a reinfecção da sífilis o não tratamento dos parceiros ${ }^{13,14,17,18}$. Tais pesquisas analisaram o conhecimento dos profissionais pré-natalistas e a qualidade da assistência pré-natal, ressaltando que a magnitude do tema ainda não se tornou visível para os profissionais de saúde que tratam de mulheres com sífilis. Além do não tratamento do parceiro, em alguns registros eles nem são mencionados.

Outro estudo ${ }^{21}$ acrescenta a reflexão de que é necessária especial atenção aos profissionais que realizam o pré-natal, oferecendo-lhes a devida capacitação e monitoramento das atividades desenvolvidas e associadas à prevenção da sífilis congênita. É necessária a busca de estratégias visando integrar o parceiro ao período gravídico.

\section{Assistência de enfermagem prestada à gestante: inadequação}

A assistência prestada no tratamento da sífilis congênita foi abordada pela totalidade das publicações. A eficácia do tratamento adequado é comprovada num estudo de caso, em que um recém-nascido, apresentando sinais de sífilis congênita, fez o teste de sorologia inicial e o tratamento adequado foi iniciado, com penicilina cristalina por um período de 10 dias. Foi acompanhado em consulta interdisciplinar com especialistas em doenças infecciosas pediátricas. Como esperado, as titulações dos exames para sífilis foram decrescendo e a criança recebeu alta, estável e próspera, com 38 semanas de idade ${ }^{16}$. Tal relato mostra que a prevenção, tratamento adequado e controle da sífilis são possíveis. Torna-se essencial a avaliação da qualidade da assistência pré-natal que vem sendo prestada nas unidades de saúde.

Um estudo de revisão bibliográfica ${ }^{12}$ constatou falha no atendimento pré-natal e dificuldade de acesso a ele. Além do tratamento inadequado, inclui o déficit de informações sobre as consequências da sífilis para o concepto e medidas profiláticas da sífilis congênita.

Outro estudo, realizado no Município de Araguari - MG, exemplifica uma estratégia que tem trazido bons resultados. Agentes comunitários de saúde identificam mulheres com atraso menstrual na comunidade e imediatamente agendam a primeira consulta para um possível diagnóstico de gravidez. Isso agiliza os exames, identifica precocemente os problemas e acelera os tratamentos pertinentes ${ }^{22}$. Este trabalho de captação destas gestantes faz parte das diretrizes do Programa Estratégia Saúde da Família.

Certas publicações, no período pesquisado, constataram que gestantes com sorologia positiva para sífilis não são tratadas ou o fazem de forma inadequada, os parceiros não são tratados e, consequentemente, elevados números de mulheres que fizeram pré-natal recebem o diagnóstico de sífilis na hora do parto $^{11-15,17,18}$.
Situações que colaboram para a baixa qualidade na assistência são referidas em pesquisas no Ceará ${ }^{14}$ e no Rio de Janeiro ${ }^{17}$ : gestantes não voltam para pegar resultado de exames; parceiros não são tratados; ocorre demora do resultado; falta acesso ao exame treponêmico; muitas maternidades não oferecem o exame sorológico como rotina para as parturientes, voltando a mãe e o bebê para a comunidade com a possibilidade de estarem com sífilis.

É constatada, também, a falha no acompanhamento das crianças tratadas com sífilis congênita. Um estudo exploratório analisou nove casos de sífilis congênita, no ano de 2010, no Ceará, e constatou que apenas uma realizou seguimento após tratamento, duas começaram e não terminaram ${ }^{13}$.

Para o alcance da meta proposta pelo Ministério da Saúde para a erradicação da sífilis, são imprescindíveis melhorias na qualidade da vigilância epidemiológica, associadas à correta aplicação das condutas preconizadas para o atendimento de gestantes e recém-nascidos acometidos pela doença. No período de 2007 a 2010, no Rio Grande do Norte, mais de dois terços dos casos de sífilis congênita ocorreram a partir de mães que haviam realizado pré-natal, não justificando tal ocorrência ${ }^{11}$. Recorrendo a contribuição de um estudo das representações sociais de gestantes que fazem o pré-natal, tem-se a constatação de que o pré-natal significa um momento primordial para a identificação de agravos que colocam em risco a saúde materna e do concepto ${ }^{21}$. O profissional de saúde, bem como toda a equipe multiprofissional, deve encontrar espaço para a discussão e orientação dos casos, com foco em um processo de cuidar dedicado aos aspectos biológicos, psicológicos e culturais da gestante. Aos profissionais envolvidos compete o compartilhamento de responsabilidades sobre o atendimento prestado no período pré-natal.

\section{Dificuldades profissionais na prevenção e controle da sífilis}

As dificuldades dos profissionais de saúde na prevenção e controle da sífilis são avaliadas por quase a totalidade das publicações ${ }^{11-14,16-18}$.

Um estudo epidemiológico, analisando a sífilis congênita na década de 2000 a 2009, no Ceará, destaca que os profissionais de saúde não estão priorizando a sífilis congênita como um problema de saúde pública; as medidas de prevenção estão sendo ignoradas; constata-se a ausência de uniformidade na conduta para o diagnóstico e tratamento da sífilis; ocorrem falhas no mecanismo de notificação e nos registros em prontuários, faltando informações importantes ${ }^{14}$. Contribui para esta temática outro estudo, realizado, também, no Ceará, em 12 centros de saúde da família, em 2009, o qual reforça que o profissional, para receber a gestante, deve estar devidamente qualificado, tendo um olhar para além da natureza física, atento a fatores 
emocionais, econômicos e familiares, pois estes podem ter influência na adesão à consulta pré-natal e, consequentemente, na qualidade do acompanhamento ${ }^{23}$.

Entre as oito publicações ${ }^{11-18}$, dois estudos exploratórios avaliam, especificamente, o conhecimento e a atuação dos profissionais de saúde no manejo da sífilis ${ }^{17,18}$.

Um estudo foi realizado no Rio de Janeiro, com 102 pré-natalistas da rede SUS, representando 70\% da amostra. Os profissionais apresentavam conhecimento correto das formas de transmissão da sífilis e afirmavam: ter participado de treinamentos sobre manejo da sífilis na gravidez nos últimos cinco anos; conhecer e aprovar os relatórios de investigação de sífilis congênita. Por outro lado, verificou-se que os mesmos profissionais revelavam: conduta inadequada em relação aos parceiros, ou não abordando ou enviando pedido de exames ou tratamento por meio da gestante; barreiras relacionadas ao conhecimento e à familiaridade com o conteúdo dos protocolos assistenciais; dificuldades na abordagem das ISTs ${ }^{17}$.

O outro estudo foi realizado com 515 profissionais da estratégia saúde da família, no Ceará Constatou a existência de profissionais de saúde que não apresentam conhecimento satisfatório sobre as medidas recomendadas pelo Ministério da Saúde para a prevenção e controle da sífilis congênita. Os profissionais demonstraram falta de conhecimento sobre o diagnóstico, tratamento e acompanhamento de gestantes com resultados positivos para sífilis. Elevada percentagem dos profissionais não conseguiu identificar corretamente a recomendação dos testes treponêmicos e não treponêmicos; $21,9 \%$ dos médicos relataram que iriam tratar as mulheres grávidas que são alérgicas à penicilina inadequadamente; 56,7\% dos profissionais revelaram falta de conhecimento sobre o tratamento para as mulheres grávidas no estágio secundário da sífilis; mais da metade deles nunca haviam sido submetidos a treinamento relacionado à sífilis ${ }^{18}$.

Resultados semelhantes foram obtidos em estudo epidemiológico realizado em Natal, no período de 2008 a 2010, que objetivou discutir as causas de óbitos neonatais reduzíveis por adequada atenção à mulher na gestação relacionando-as à assistência de enfermagem prestada durante o pré-natal. Comprovou que a mortalidade neonatal pode estar relacionada a causas preveníveis, dificuldade de acesso ao serviço de saúde, além da falta de qualidade na assistência pré-natal, ao parto e ao recém-nascido ${ }^{24}$.

Estudo realizado no Rio Grande do Norte, no período de 2007 a 2010, descreveu a ocorrência de 598 casos de notificação de sífilis congênita e constatou falhas nos registros dos profissionais: informações ignoradas ou em branco, como a escolaridade materna, o tratamento do parceiro e a realização do pré-natal ${ }^{11}$.

Estudos no Ceará ${ }^{13,14,18}$ e Rio de Janeiro ${ }^{17}$ também conferem que os registros de casos de sífilis em mulheres grávidas e de sífilis congênita estão ainda muito abaixo do esperado, demonstrando deficiências significativas na qualidade das informações por falha nos registros.

Lacunas no conhecimento dos profissionais, quanto à gestão da sífilis na gravidez, podem comprometer a eficácia da prevenção e controle da sífilis congênita. Faz-se necessário uma especial atenção aos profissionais que realizam o pré-natal, oferecendo-lhes a devida capacitação.

É de extrema importância que os profissionais de saúde realizem consulta de pré-natal de gestante com sífilis de forma correta e de qualidade. A política pública de saúde deve estabelecer estratégias junto aos profissionais de saúde para evitar a transmissão da sífilis ${ }^{12}$.

\section{Conclusão}

O presente artigo analisou e discutiu publicações científicas, relacionada à sífilis congênita e a enfermagem, no período de 2005 a 2014. Concluiu-se que o número de publicações da enfermagem envolvendo o tema é reduzido. Utilizam métodos como os documentais, estudo de caso, revisão bibliográfica, estudo avaliativo do conhecimento e da prática. $\mathrm{O}$ enfoque dos artigos está voltado para a patologia, tratamento, sequelas, diagnóstico diferencial, conhecimento, fatores de risco e fatores sociais.

A limitação do estudo consiste em ter artigos selecionados com metodologias distintas, fato que impede a comparação dos dados e impossibilita a generalização dos resultados.

A análise dos artigos traz reflexões importantes, principalmente, no que tange à assistência prestada no pré-natal. Identifica fatores de risco que torna a mulher vulnerável à sífilis, constata a inadequação da assistência prestada e elenca dificuldades no combate à transmissão vertical da sífilis relacionada à falta de capacitação profissional no rastreamento, diagnóstico e condução do tratamento da gestante com sífilis.

A sífilis na gestação e a sífilis congênita atingiram proporções contrárias ao que foi proposto pelo Ministério da Saúde. Continuam sendo um desafio à prevenção e ao controle da doença.

Sugere-se que os resultados trazidos neste estudo sejam confrontados com a prática das várias unidades que prestam assistência pré-natal. $\mathrm{O}$ impacto das informações aqui fornecidas poderá ajudar a formular ações que visem à melhoria da qualidade da atuação do profissional.

\section{REFERÊNCIAS}

1.Geraldes Neto B, Soler ZASG, Braile DM, Daher W. A sífilis no século XVI: o impacto de uma nova doença. Arq Ciência e Saúde. 2009; 16(3):127-9.

2.Silva FFA. Sífilis e HIV na gestação: uma investigação 
sobre a assistência pré-natal na rede SUS do município do Rio de Janeiro 2007/2008. [dissertação de mestrado]. Rio de Janeiro: Escola Nacional de Saúde Pública; 2010. 3.Becker MEL. Avaliação da qualidade da assistência pré-natal na atenção primária no município de Rio Branco - Acre [dissertação de mestrado]. Rio de Janeiro: Escola Nacional de Saúde Pública; 2011.

4.Azulay RD. História da sífilis. Rev Bras Dermatol.1988; 63(1):3-4.

5.Avelleira JCR, Bottino G. Sífilis: diagnóstico, tratamento e controle. Educação Médica Continuada. Rev Bras Dermatol. 2006; 81(2):111-26.

6.Ministério da Saúde (Br). Programa Nacional de DST/ AIDS/ Hepatites Virais. Boletim Epidemiológico - sífilis 2012. Brasília (DF): Secretaria de Vigilância em Saúde; 2012.

7.Ministério da Saúde (Br). Transmissão vertical do HIV e sífilis: estratégias para redução e eliminação. Brasília (DF): Editora MS; 2014.

8.Souza MT, Silva MD, Carvalho R. Revisão integrativa: o que é e como fazer. Einstein. 2010; 8(1):102-6.

9.Mendes KDS, Silveira RCCP, Galvão CM. Revisão integrativa: método de pesquisa para a incorporação de evidências na saúde e na enfermagem. Texto contexto enferm. 2008; 17:758-64.

10.Botelho LLR, Cunha CCA, Macedo M. O método da revisão integrativa nos estudos organizacionais. Rev eletr Gestão Soc. 2011; 5(11):121-36.

11.Carvalho IS, Brito RS. A sífilis congênita no Rio Grande do Norte: estudo descritivo do período 2007-2010. Epidemiologia e serviços de saúde. 2014; 23:287-94.

12.Albuquerque GMA, Chaves EMC, Sampaio LRL, Dias KCF, Patrocínio MCA, Vasconcelos SMM. Complicações da sífilis congênita: uma revisão de literatura. Pediatria Moderna. 2014; 50:254-8.

13.Mesquita KO, Lima GK, Filgueira AA, Flôr SMC, Freitas CASL, Linhares MSC, et al. Análise dos casos de sífilis congênita em Sobral, Ceará: contribuições para assistência pré-natal. DST - J bras Doenças Sex Transm. 2012; 24(1):20-7.

14.Costa CC,Freitas LV,Sousa DMN,Oliveira LL,Chagas
ACMA,Lopes MVO, et al. Sífilis congênita no Ceará: análise epidemiológica de uma década. Rev esc enferm USP. 2013; 47:152-9

15.Grossi R, Barros AC, Andrade R, Galvão C, Reis $\mathrm{H}$, Ferreira DC et al. Reação de Jarisch-Herxheimer em gestante com sífilis: relato de caso. DST - J bras Doenças Sex Transm. 2011; 23:149-51.

16.Follett T, Clarke D. Resurgence of congenital syphilis: diagnosis and treatment. Neonatal Netw. 2011; 30:320-8.

17.Soares RM, Domingues M, Lauria LM, Saraceni V, Leal MC. Manejo da sífilis na gestação: conhecimentos, práticas e atitudes dos profissionais pré-natalistas da rede SUS do município do Rio de Janeiro. Ciência e saúde coletiva. 2013; 18:1341-51.

18.Silva DMA, Araújo MAL, Silva RM, Andrade RFV, Moura HJ, Esteves ABB. Knowledge of healthcare professionals regarding the vertical transmission of syphilis in Fortaleza-CE, Brazil. Texto contexto - enferm. 2014; 23:278-85.

19. Holanda MTCG, Barreto MA, Machado KMM, Pereira RC. Perfil epidemiológico da sífilis congênita no município de Natal, Rio Grande do Norte - 2004 a 2007. Epidemiol Serv Saúde. 2011; 20:203-12.

20.Domingos AC. Gravidez na adolescência: enfrentamento na estratégia de saúde da família [trabalho de conclusão de curso]. Belo Horizonte (MG): Universidade Federal de Minas Gerais; 2010.

21.Duarte SJH. Motivos que levam as gestantes a fazerem o pré-natal: um estudo das representações sociais. Cienc Enferm. 2012; 18(2):75-82.

22.Miranda FJS, Fernandes RAQ. Assistência pré-natal: estudo de três indicadores. Rev enferm UERJ. 2010; 18:179-84.

23.Peixoto CR, Freitas LV, Teles LMR, Campos FC, Paula PF, Damasceno AKC. O pré-natal na atenção primária: o ponto de partida para reorganização da assistência obstétrica. Rev enferm UERJ. 2011; 19:286-91. 24.Brandão ICA, Godeiro ALS, Monteiro AI. Assistência de enfermagem no pré-natal e evitabilidade de óbitos neonatais. Rev enferm UERJ. 2012; 20:596-602. 